\title{
Lesson of the month 2: Autoimmune sequelae of anti-GAD antibodies - thinking outside the box
}

\author{
Authors: Rebecca J Ward, ${ }^{A}$ George I Varughese, ${ }^{B}$ Biju Jose ${ }^{B}$ and Roby J Abraham ${ }^{C}$
}

\begin{abstract}
A 52 year-old female with no significant medical problems presented with left-sided weakness, unsteady gait and speech disturbance. It was thought that she had neuro-inflammation and she remained clinically stable. Several years later, she was diagnosed with latent autoimmune diabetes of adulthood. Her neurological symptoms deteriorated and she was admitted into hospital. The cerebrospinal fluid was normal, as were an array of blood tests. Imaging tests, including magnetic resonance imaging, computerised tomography and positron emission tomography scans were normal. However, her anti-glutamic acid decarboxylase antibody serum level, which had been taken in the diabetes outpatient clinic, returned at 2,000,000 IU/mL (normal range $0-10$ ). This led to the diagnosis of glutamic acid decarboxylase (GAD) positive cerebellar ataxia. She was treated with plasma exchange and intravenous immunoglobulins and over next 12 weeks her symptoms improved. Our case highlights the need for appropriate treatment of patients with GAD positive cerebellar ataxia to achieve good outcomes.
\end{abstract}

KEYWORDS: Anti-GAD, ataxia, autoimmune, diabetes, glutamic acid decarboxylase antibodies

\section{Introduction}

Autoimmune causes of central nervous system disorders are being increasingly recognised as important; potentially reversible non-infectious causes and several case studies have been reported for this rarer cause of encephalitis. ${ }^{1-3}$ Autoimmune encephalitis symptoms may include cognitive impairment, psychiatric manifestations, abnormal movements and seizures. ${ }^{4}$ However, if the involvement is predominantly in the cerebellum then it is described as autoimmune cerebellar ataxia and patients present with cerebellar signs and symptoms without high mental function involvement. Because of the wide range of the presenting symptoms, numerous investigations are often performed before a conclusive diagnosis of cerebellar ataxia is

Authors: ${ }^{\text {A }}$ academic foundation trainee, University Hospitals of North Midlands NHS Trust, Stoke-on-Trent, UK; ${ }^{\mathrm{B}}$ consultant in diabetes and endocrinology, University Hospitals of North Midlands NHS Trust, Stoke-on-Trent, UK; ${ }^{C}$ Consultant neurologist, University Hospitals of North Midlands NHS Trust, Stoke-on-Trent, UK made. This may lead to a delay in diagnosis and treatment, which may affect prognosis. Often, it is difficult to clinically distinguish between autoimmune and infectious causes of central nervous system disorders. In this report, we summarise the case of a woman who presented with a myriad of symptoms that remained undiagnosed until her development of latent autoimmune diabetes of adulthood (LADA) clinched the diagnosis.

\section{Case history}

A 52-year-old right-handed female manager presented with left-sided limb weakness, unsteady gait and speech disturbance. Magnetic resonance imaging (MRI) brain and whole spinal cord showed white matter changes but these were not typical for demyelination. It was thought that she had neuro-inflammation.

She remained clinically stable and had a repeat MRI scan 2 years later, which showed no significant changes. She reported slight improvement in symptoms but could only walk 500 yards. She was diagnosed with diabetes 4 years after her initial presentation; there was debate whether it was type 1 or type 2 diabetes because of her age and the acute onset. Therefore, further tests - including GAD (glutamic acid decarboxyglase) antibodies, anti-insulin antibodies and antiislet antibodies - were requested.

A few months later at the neurology follow-up clinic she reported severe headaches and progressive unsteadiness. She was ataxic, unable to stand, had cerebellar and bulbar dysarthria, severe intention tremor, brisk deep tendon reflexes and finger-nose and heel-shin ataxias, but a normal plantar response. Her left plantar reflex was extensor response. She was admitted urgently to the neurology ward.

MRI brain and whole spinal cord demonstrated white matter T2 hyperintense lesions in the brain and excluded cerebrovascular disease. Lumbar puncture showed normal protein $(0.53 \mathrm{~g} / \mathrm{L})$ and glucose $(3.4 \mathrm{mmol} / \mathrm{L})$. The cerebrospinal fluid (CSF) was acellular. Oligoclonal band and CSF serology were negative. Serum copper, ceruloplasmin, alpha-fetoprotein, folate, vitamin B12, thyroid function, immunoglobulin profile, coeliac screen, paraneoplastic antineuronal and antipurkinje cell antibodies, and blood film were all normal. computerised tomography chest/abdomen/pelvis and positron emission tomography scans to investigate possible malignancy were normal. However, the anti-GAD antibody level in blood returned at 2,000,000 $\mathrm{IU} / \mathrm{mL}$ (normal range $0-10 \mathrm{IU} / \mathrm{mL}$ ) and subsequently anti-GAD antibody level in the CSF was 
$28,000 \mathrm{IU} / \mathrm{mL}$. In view of significantly high titre of anti-GAD antibodies, GAD positive cerebellar ataxia was suspected.

She was treated with plasma exchange and intravenous immunoglobulins (IVIG). Over 12 weeks her speech, gait and truncal ataxia improved. She received a 5-day course of 6-weekly IVIG (Privigen, CSL Behring AG, Bern, Switzerland) following her admission and her symptoms improved and remained stable. She has a predominantly ataxic syndrome needing a walking stick and wheelchair for long distances. She does not have memory deficits or episodes of higher mental function symptoms, which corresponds with a diagnosis of cerebellar ataxia rather than autoimmune encephalitis.

\section{Discussion}

GAD antibodies have been associated with several neurological disorders, including stiff person syndrome - which is when patients present with muscle stiffness and is the commonest of the GAD-positive neurological syndromes; cerebellar ataxia is the second commonest and autoimmune encephalitis, when there is high mental function involvement, is the third. GAD antibodies have also been associated with paraneoplastic encephalitis and this highlights the need for extensive investigation to rule out tumours. Additionally, anti-GAD antibodies are seen in autoimmune forms of diabetes, such as type 1 diabetes mellitus and late onset diabetes mellitus, ${ }^{5}$ also known as LADA. However, levels of the antibody are often much lower than those seen in cases with encephalitis. Such high levels of GAD antibodies tend to correlate with neurological syndromes. ${ }^{5}$

Given the rarity of autoimmune encephalopathic syndromes, patients are often tested and treated for infective causes of encephalitis. A thorough history should be taken to determine medical, travel and sexual history to consider risk factors for autoimmune and infectious causes of encephalitis. Other conditions that can present similarly include multiple sclerosis (which may be diagnosed with imaging and lumbar puncture), acute disseminated encephalomyelitis (which again has characteristic brain imaging findings), and lupus, which can present with neuropathy, vasculitis and stroke.

There is debate regarding the most appropriate treatment for anti-GAD encephalitis. Malter et al found treatment with monthly methylprednisolone did not help symptoms in nine patients who presented with anti-GAD encephalitis. ${ }^{6}$ Arino et al looked at the long-term follow-up (median: 5.4 years) of patients with cerebellar ataxia. They found that patients with a subacute presentation were more likely to respond to immunotherapy, including IVIG and corticosteroids, and show a clinical improvement. ${ }^{7}$ Other case reports have shown clinical improvement with IVIG and plasma exchange. ${ }^{2,8,9}$ However, as far as the authors are aware, there are no large randomised controlled trials looking at the effectiveness of treatment for patients with anti-GAD encephalopathy. However, this patient responded well to regular IVIG treatment.

\section{Conclusion}

We conclude that if GAD positive cerebellar ataxia is diagnosed and treated appropriately (including aggressive treatment with therapies such as high-dose steroids, IVIG, plasma exchange, rituximab and cyclophosphamide), symptoms may be able to be reversed and further progression halted.
Patients presenting with this constellation of symptoms should undergo an assessment with CSF studies, an autoimmune screen, brain MRI, electroencephalogram and a full-body computerised tomography scan. It took 4 years from onset of symptoms before a diagnosis was reached. In fact, it was the diagnosis of LADA that gave the diagnosis away. This emphasises the need for referral between specialties as this patient was cared for under the endocrinologists and neurologists. The patient reported symptomatic improvement after treatment with IVIG.

This case highlights the difficulties of diagnosing GADpositive cerebellar ataxia, which is recognised but less commonly perceived in routine clinical practice.

\section{Conflict of interest}

RJA has received educational support towards conference expenses from Novartis, Sanofi Genzyme and Biogen. BJ has received educational support towards conference expenses from Sanofi. All authors declare no other conflicts of interest.

\section{Consent}

Written consent was obtained from the patient for the publication of the clinical details in this article.

\section{Acknowledgements}

The authors would like to than Dr Natarajan, a stroke consultant who provided initial support, and Dr Harimam, a specialty registrar who presented this case as an abstract at the Society of Endocrinology BES conference.

\section{References}

1 Finelli PF. Autoimmune limbic encephalitis with GAD antibodies. Neurohospitalist 2011;1:178-81.

2 Incecik F, Hergüner O, Yıldızda D, Horoz O, Besen S. Limbic encephalitis with antibodies to glutamic acid decarboxylase presenting with brainstem symptoms. Ann Indian Acad Neurol 2015;18:243-5.

3 Sharma A, Dubey D, Sawhney A, Janga K. GAD65 Positive autoimmune limbic encephalitis: a case report and review of literature. J Clin Med Res 2012;4:424-8.

4 Lancaster E. The diagnosis and treatment of autoimmune encephalitis. J Clin Neurol 2016;12:1-13.

5 Honnorat J, Saiz A, Giometto B et al. Cerebellar ataxia with antiglutamic acid decarboxylase antibodies: study of 14 patients. Arch Neurol 2001;58:225-30.

6 Malter MP, Helmstaedter C, Urbach H, Vincent A, Bien CG. Antibodies to glutamic acid decarboxylase define a form of limbic encephalitis. Ann Neurol 2010;67:470-8.

7 Arino H, Gresa-Arribas N, Blanco Y et al. Cerebellar ataxia and glutamic acid decarboxylaseantibodies. Immunologic profile and long-term effect of immunotherapy. JAMA Neurol 2014;71:1009-16.

8 Korff CM, Parvex P, Cimasoni L et al. Encephalitis associated with glutamic acid decarboxylase autoantibodies in a child: a treatable condition? Arch Neurol 2011;68:1065-8.

9 Mazzi G, Roia DD, Cruciatti B, Matà S, Catapano R. Plasma exchange for anti-GAD associated non-paraneoplastic limbic encephalitis. Transfus Apher Sci 2008;39:229-33.

Address for correspondence: Dr George Varughese, Department of Diabetes and Endocrinology, Royal Stoke University Hospital, Stoke-on-Trent ST4 6QG, UK. Email: george.varughese@uhnm.nhs.uk 